\title{
Pratiques
}

Linguistique, littérature, didactique

145-146 | 2010

Didactique du français (1)

\section{« Finançons les études littéraires »}

Extrait de la table ronde

\section{Raymond Michel}

\section{(2) OpenEdition}

Journals

\section{Édition électronique}

URL : http://journals.openedition.org/pratiques/1492

DOI : 10.4000/pratiques. 1492

ISSN : 2425-2042

Éditeur

Centre de recherche sur les médiations (CREM)

\section{Édition imprimée}

Date de publication : 15 juin 2010

Pagination : 33-38

\section{Référence électronique}

Raymond Michel, « «Finançons les études littéraires » », Pratiques [En ligne], 145-146 | 2010, mis en ligne le 15 juin 2010, consulté le 02 mai 2019. URL : http://journals.openedition.org/pratiques/1492 DOI : 10.4000/pratiques. 1492 


\section{« Finançons les études littéraires »}

Il n'est pas aisé, aujourd'hui - mais cela a, peut-être, sinon sûrement, été toujours le cas - de parler de littérature, et plus précisément d'études littéraires, tant elles sont considérées, à notre époque, comme peu légitimes que ce soit au regard des champs scientifiques et techniques qu'au regard des exigences de rentabilité d'une société libérale, vouée à la production et à la circulation de richesses marchandes. Pour s'en convaincre, il suffit de se souvenir du véritable feuilleton qu'ont produit les démêlés de Nicolas Sarkozy ${ }^{(1)}$ avec La Princesse de Clèves et avec « la littérature ancienne».

Il ne s'agit pas seulement du peu d'estime que, au plus haut niveau de l'État, on porte aux études littéraires, car une telle délégétimation rejoint d'autres phénomènes qui vont dans le même sens : la dévaluation des études littéraires tant dans le secondaire (la série L, au lycée, semble réservée, à quelques exceptions près, aux élèves qui ne peuvent suivre un cursus en S) que dans le supérieur (voir le peu d'engouement des étudiants pour entreprendre des études de lettres et le choix par défaut d'une grande partie de ceux qui les suivent) ${ }^{(2)}$. Une telle situation pourrait expliquer la légitime morosité qui gagnerait le didacticien en littérature, acculé à défendre sont territoire et son gagne-pain - morosité qui fait écho à l'ennui que connaissent certains élèves et certains étudiants pendant un cours de littérature. Mais ce dernier peut, aussi, passer à l'offensive, comme l'y invite le livre d'Yves Citton, Lire, interpréter, actualiser. Pourquoi les études littéraires ${ }^{(3)}$. Ce livre a pour ambition, entre autres, « de comprendre àquoi peuvent servir les études littéraires au sein des évolutions actuelles de nos formes sociales » et nous invite à « envisager ce que même les barbares pourraient gagner à lire La Princesse de Clèves»

Il me semble que cet ouvrage mériterait une diffusion et une discussion élargie au sein de toux ceux qui se préoccupent des études littéraires, tant il ouvre des horizons nouveaux dans l'approche de la littérature. C'est ce que je voudrais faire, ici, très succinctement, en prenant le risque de trahir et de simplifier quelque peu la richesse de la réflexion d'Yves Citton. J'évoquerai, d'abord, le cadre général de la réflexion d'Yves Citton, puis ses enjeux politiques, et enfin quelques pistes didactiques qu'impliquent ses prises de position.

\section{Un plaidoyer pour les lectures actualisantes}

Au-delà de sa force revigorante et optimiste, cet ouvrage présente de multiples intérêts pour toute personne qui est soucieux d'herméneutique littéraire et de sa didactique. Pour aller à l'essentiel, dans le cadre de cette réflexion, j'aimerais en dégager deux : 1/ Un élargissement salutaire des références théoriques qui fondent les études littéraires ; 2/ La mise en perspective théorique et pratique d'une notion centrale, la lecture actualisante.

(1) Voir : http ://passouline.blog.lemonde.fr/2008/04/16/le-president-veut-il-la-peau-de-laprincesse/.

(2) La nouvelle mouture des Capes de lettres modernes et de lettre classiques, élaborée dans le cadre de la mastérisation des métiers de l'enseignement, montre à quel point les finalités et les objectifs des études littéraires sont floues - pour rester dans l'euphémisme - et pourrait fonder, à juste titre, l'impression que l'on aurait qu' " elles ne servent pas à grand chose ».

(3) Yves Citton, Lire, interpréter, actualiser. Pourquoi les études littéraires?, Paris, Éditions Amsterdam, 2007. Sauf avis contraire, les italiques dans les citations de l'ouvrage que je serai amené à faire sont toujours de l'auteur. 
Yves Citton entreprend, tout d'abord, une véritable déconstruction des vielles lunes - bien connues, mais qui persistent sous des oripeaux plus ou moins subtils, plus ou moins « modernes » et « scientistes »-qui entravent de fait tout travail herméneutique engageant et passionnant. Je cite, pour mémoire et sans souci d'exhaustivité et de hiérarchie : l'hégémonie toujours recommencée de l'histoire littéraire ; l'intangibilité du texte et son antériorité sur sa lecture ; le « sens » caché d'une œuvre qu'il faudrait retrouver au-delà de la surface du texte ; la sujétion et le recours en dernière instance à l'intention (consciente ou non) de l'auteur ; le littéralisme obligatoire couplé à l'étude « objective » des structures textuelles; le choix hypostasié du générique contre la singularité de l'œuvre conçue, alors, comme pure échantillon de sa classe ; la forclusion du corps et de la subjectivité du sujet lisant, etc.

Pour rompre avec ces présupposés qui ne peuvent conduire qu'à des apories irréductibles, Yves Citton s'appuie sur trois courants de pensée, qu'il concilie sans problème : d'une part, le pragmatisme américain (Stanley Fish, Richard Rorty...) ; d'autre part, la galaxie du néospinozisme des multitudes (Paolo Virno, Gilles Deleuze, Gilbert Simondon, Laurent Bove, Antonio Negri...) ; et, enfin, l'ontologie d'Alain Badiou. Il faudrait citer, aussi, des auteurs comme Luis Prieto, Jacques Rancière, Jean-Marie Schaeffer, Thomas Pavel, Dominique Maingueneau, Iouri Lotman, Laurent Jenny, Marc Escola, Lubomir Dolezel, Jean Baudrillard, Mikhaïl Bakhtine... Une telle liste, dont on pardonnera le caractère très sommaire et l'effet de fouillis qu'elle produit, n'a que l'ambition d'exhiber les référents théoriques d'Yves Citton et d'insister sur la pluralité des champs qu'il convoque (philosophie, linguistique, sociologie, anthropologie...). Cet éclectisme théorique est revendiqué, explicitement, par l'auteur, puisque selon lui toute condition de pensée et toute conviction s'inscrivent dans une con-diction: «je ne parle qu'avec la voix d'un autre ${ }^{(4)} »$. Yves Citton substitue aux approches « traditionnelles », fondées sur une conception causaliste et substantielle de la signification d'un texte littéraire - toujours déjà-là, figée en amont, mais cependant cachée et tue -, les puissances de l'interprétation active produite par les jeux collectifs de l'interlocution littéraire. La lecture est pensée, ici, comme une activité pleine et entière. En effet, Yves Citton montre avec beaucoup d'à propos comment le lecteur est amené à projeter ses préconceptions et ses préjugés sur l'œuvre qu'il est en train de lire.

Ces jeux de projection interprétative ${ }^{(5)}$ - il s'agirait donc moins de lire des textes littéraires que de lire littérairement des textes - ne trahissent pas le « sens " (authentique, originel, objectif) d'une œuvre, et la « littérarité » d'un texte ne dépend pas de marqueurs (linguistiques, textuels, ou sémiotiques) indépendants de tout choix, mais « d'une projection opérée par le lecteur [...] qui accommode le matériau textuel conformément à une certaine "recette de cuisine", en sorte qu'il satisfasse le goût que nous avons développé pour la littérature ${ }^{(6)}$. ». Mais cette projection n'est en rien

(4) Yves Citton, Lire, interpréter, actualiser. Pourquoi les études littéraires?, op. cit., p. 311.

(5) Yves Citton exploite à ce propos la fable expérimentale racontée par Stanley Fish, dans Quand lire, c'est faire. L'autorité des communautés interprétatives, trad. D'E. Dobenesque, Paris, Les Prairies ordinaires, 2007. Le professeur Fish enseigne dans la même classe deux cours consécutifs, l'un sur la théorie littéraire, l'autre sur la poésie littéraire anglaise $\mathrm{du} 17^{\mathrm{e}} \mathrm{s}$. À la fin du premier cours, il avait écrit, sur le tableau, en guise de bibliographie à lire, une petite liste de noms de linguistes et de critiques ; après avoir encadré cette bibliographie, il présente au deuxième groupe cette liste comme un poème religieux du type de ceux qu'ils avaient déjà étudiés et leur demande de l'interpréter comme tel. La machine à interpréter se met en route, et les étudiants font preuve d'imagination pour rendre cohérent ce « poème». Ce qui amène Stanley Fish à conclure que «l'interprétation n'est pas un art de la compréhension, mais de la construction. Les interprètes ne décodent pas les poèmes : ils les font » (Je souligne).

(6) Yves Citton, Lire, interpréter, actualiser. Pourquoi les études littéraires ?, op. cit., p. 63. 
purement subjective et solipsiste ; car Stanley Fish montre que tout lecteur appartient à une (ou des) communauté(s) interprétative(s) qui structurent et normalisent les compétences herméneutiques de l'interprète. Il n'y a donc pas, à proprement parler, d'interprétation « fausse » par rapport à un sens « objectif» du texte, mais des interprétations plus ou moins acceptables au sein de la communauté interprétative dans laquelle se tient le lecteur.

\section{Enjeux politiques du travail herméneutique}

L'ouvrage d'Yves Citton a le mérite de montrer, aussi, qu'il nous est possible de concilier actualisation socio-politique et «belle-lettrisme», tout en nous donnant les moyens théoriques d'habiter au mieux cet entre-deux. En effet, il existe une politique de la littérature, qui, comme le signale Jacques Rancière, ne concerne ni « la politique des écrivains [...], leurs engagements personnels dans les luttes politiques de leur temps », ni « la manière dont ils représentent dans leurs livres les structures sociales, les mouvements politiques ou les identités diverses ». Car,

[1'] expression « politique de la littérature » implique que la littérature fait de la politique en tant que littérature [...] dans ce découpage des espaces et des temps, du visible et de l'invisible, de la parole et du bruit. Elle intervient dans ce rapport entre des pratiques, des formes de visibilité et des modes du dire qui découpe un ou des mondes communs ${ }^{(7)}$.

Les réflexions d'Yves Citton, on le voit, entrent directement en écho avec les préoccupations de l'auteur du Partage du sensible ${ }^{(8)}$. Aussi me paraît-il utile de signaler, rapidement, sinon caricaturalement, quelques enjeux politiques, au sens rancièrien de ce terme, pointés par Yves Citton, qui justifient qu'il faille financer les études littéraires « si nous voulons mieux comprendre les modèles d'individuation et de socialisation qui régissent notre devenir, et si nous voulons permettre à nos formations sociales de produire des sujets capables de se donner des valeurs épanouissantes et réfléchies ${ }^{(9)}$. $»$

\section{Une autonomie critique}

Une telle conception de l'activité herméneutique, prônée Yves Citton, permet aux lecteurs de retrouver une certaine autonomie critique. Ceux-ci, dès lors, peuvent envisager de prendre leurs distances et de rompre avec tous les " gardiens du sens », attachés à la monovalence et à l'objectivité scientiste. Cette autonomie a pour corollaire l'indépendance énonciative, à savoir la capacité pour chacun de pouvoir poser « la question du sens des textes » et de «s'emparer du pouvoir de poser les questions qui comptent, plutôt [que de] se contenter de répondre à celles qu'aura formulées autrui (en fonction de ses pertinences propres) ${ }^{(10)}$. » En effet, lire littérairement un texte passe par une sensibilisation exacerbée aux propriétés connotatives de tout écrit.

Le travail littéraire - auquel, insiste Yves Citton, participent conjointement l'auteur et l'interprète, ce qui explique qu'il faille parler d'interlocution littéraire - est donc propice à une reconfiguration disruptive de nos fins et de nos priorités et à une prise de distance critique - du fait même de la confrontation avec l'autre qu'est le texte - par rapport au vocabulaire final ${ }^{(11)}$ dont nous héritons sans nous en rendre

(7) Jacques Rancière, Politique de la littérature, Paris, Galilée, 2007, pp. 11 et 12.

(8) Jacques Rancière, Le Partage du sensible. Esthétique et politique, Paris, La Fabrique, 2000.

(9) Yves Citton, Lire, interpréter, actualiser. Pourquoi les études littéraires?, op. cit., p. 304.

(10) Ibid., p. 217.

(11) «Vocabulaire final : terme forgé par Richard Rorty pour désigner, au sein d'un vocabulaire, 
compte. Aussi l'interlocution littéraire constitue-t-elle « un site d'expérimentation et de négociation unique pour mesurer et gérer la pluralité linguistique et axiologique du monde qui nous entoure et qui nous constitue ${ }^{(12)}$. »

\section{Des processus de subjectivation inédits contre tous les fondamentalismes}

Interpréter un texte c'est aussi vivre une alternance de subjectivations et de désubjectivations au cours desquelles chaque lecteur se construit et tente de rendre son monde habitable. C'est dans ce sens qu'il faut comprendre la Maxime 136 de La Rochefoucauld qu'aime citer Yves Citton : «Il y a des gens qui n'auraient jamais été amoureux, s'ils n'avaient jamais entendu parler d'amour. » Aussi Yves Citton propose-t-il de concevoir les fictions comme « des laboratoires de construction de mondes à venir » et des « usines de retraitement permanent des valeurs ${ }^{(13)}$ ». Il rejoint sur ce point les pragmatistes américains qui nomment worldmaking, cette aptitude à produire des mondes. Et sa conception n'est pas sans rappeler les remarques de Paul Ricœur qui voit dans cette " narrativité inchoative » une qualité consubstantielle de "l'homme capable », dans la mesure où chaque texte offre à notre subjectivité lectrice « un monde que [nous pourrions] habiter et dans lequel [nous pourrions] projeter [nos] pouvoirs les plus propres ${ }^{(14)} \gg$. Il est donc légitime de se poser la question des usages de la fiction, située toujours aux confins de l'imaginaire et du possible. En effet, on peut penser, avec Yves Citton, que, si la fiction a une possible fonction émancipatrice, c'est parce qu'elle :

[...] offre au lecteur l'occasion d'une délocalisation qui relève du mode utopique en ce que a) elle constitue une exercice mental sur les possibles latéraux ${ }^{(15)}$ à la réalité, et en ce que b) elle permet au lecteur d'expérimenter des réagencements affectifs capables de frayer de nouveaux possibles, en une époque où l'économie des affects devient le terrain de lutte central de nos développements sociétaux ${ }^{(16)}$.

En effet, la fiction permet ainsi de « tracer progressivement des chemins dirigeant le monde actuel vers certains de ses devenirs possibles ». Car elle « nous habitue à conjuguer une willing suspension of disbelief ${ }^{(17)}$, propre à nous rendre disponibles pour l'invention active d'un autre monde possible, avec une witty suspicion of all beliefs $^{(18)}$, qui nous encapacite à lutter contre toutes les formes de fondamentalisme

le sous-ensemble de mots qu'un locuteur ne parvient pas à définir de façon satisfaisante à 1'aide d'autres mots ; le vocabulaire final comprend donc les "valeurs" ultimes sur lesquelles reposent la philosophie ou l'idéologie de ce locuteur. »Ibid., p. 352.

(12) Ibid., p. 302.

(13) Ibid., p. 303

(14) Paul Ricœur, Temps et récit 1. L’intrigue et le récit historique, Paris, Le Seuil 1983, pp. 151-152.

(15) «Latéralisation: capacité de l'esprit humain à recombiner les informations reçues par les sens de façon à imaginer des "possibles latéraux" non réalisés, mais que les pratiques humaines peuvent faire advenir (philosophie de Raymond Ruyer). " Yves Citton, Lire, interpréter, actualiser. Pourquoi les études littéraires?, op. cit., p. 344.

(16) Ibid., p. 303.

(17) Expression « traduisible littéralement comme une "suspension volontaire de méfiance" ", proposée par Coleridge et qui désigne «l'attitude du lecteur-spectateur qui accepte momentanément de croire aux personnages et aux intrigues d'une fiction comme s'il s'agissait de personnes existantes (ce qui le conduit à éprouver des affects de peur ou d'espoir envers eux, et à neutraliser sa méfiance envers des invraisemblances qu'il ne tolérerait pas face à des faits présentés comme réels). » Ibid, p. 353.

(18) Expression « traduisible comme une "suspicion opérée par le jeu des mots envers toute croyance", qui désigne les habitudes de scepticisme que cultive en nous la pratique des fictions, en nous rendant sensibles aux vertus subversives des jeux de mots (le Wit anglais, le Witz allemand, le "bel esprit" de l'âge classique) et en nous conduisant à suspecter toute histoire prétendument véridique de relever en réalité de la fable. » Ibid., p. 353. 
- le jeu d'ensemble complexe de cette conjugaison problématique nous aidant à gérer plus prudemment nos croyances ${ }^{(19)}$.»

\section{«Scolarisation »}

Bien évidemment, et Yves Citton le revendique volontiers, ses analyses sur l'herméneutique littéraire entrent en écho avec celles de nombreux auteurs, qu'ils soient philosophes, écrivains, historiens ou sociologues. Il en est de même avec les conséquences didactiques qu'il tire de ses thèses, dans un chapitre intitulé «X Scolarisation ${ }^{(20)} »$. Il rend, d'ailleurs, volontiers, hommage aux chercheurs et aux praticiens qui ont œuvré dans ce domaine, dans la mesure où il retrouve dans leurs ouvrages des préoccupations très proches des siennes ${ }^{(21)}$. C'est pourquoi, en ce domaine, il ne faut pas penser le rapport entre la théorie et la didactique en termes d'application mécanique, mais bien en termes de dialogue constructif, qui permet à chacun de se constituer (en s'interrogeant et en interrogeant son « dehors») dans la différence de l'autre.

Il ne s'agit pas pour moi, ici, d'exposer, dans le détail les propositions d'Yves Citton, mais de relever et de cartographier - afin de les soumettre à la réflexion et à la discussion des didacticiens - les huit registres d'argumentaires qu'il sélectionne. A savoir: la motivation, la vaccination, l'élaboration, l'innovation, l'individuation, la collectivisation, la dialogisation et l'improvisation. Ceux-ci tentent de répondre à de questions qui taraudent tout didacticien de la littérature : «Pourquoi inscrire l'affabulation littéraire dans le cadre d'une école? ; " Quels avantages peut-il y avoir à scolariser une expérience qui paraît devoir relever d'une interaction singulière entre un texte et un lecteur ? "; "Ne suffit-il pas que l'école "joue un rôle premier" et qu'elle en revienne aux fondamentaux (déchiffrage des lettres et phrases (savoir lire) et des chiffres en équations (savoir compter)) ${ }^{(22)}$ ? »

Pour conclure, si l'on suit les analyses d'Yves Citton, on ne peut qu'être convaincu que les études littéraires sont loin d'être « inutiles » et que, bien au contraire, elles mériteraient que les pouvoirs publics aient le souci de les promouvoir et de les financer. Et comme l'auteur de Lire, interpréter, actualiser le dit avec force, il faut le faire :

- « si nous voulons vivre dans des collectivités de lecteurs cultivés »;

- « si nous voulons réduire l'emprise des fondamentalismes au sein de nos collectivités »;

- « si nous voulons promouvoir notre capacité à élaborer du sens selon les procédures complexes que requiert la complexité de nos formes de vies actuelles, et dont l'interprétation littéraire constitue le meilleur terrain d'exercice »;

- « si nous voulons promouvoir simultanément une source d'innovations spécifiques (produites par l'élaboration des chimères) et, ce qui n'est pas moins précieux, une gymnastique mentale qui entraîne l'esprit à la pratique de l'innovation »;

(19) Ibid., p. 303.

(20) Yves Citton, Lire, interpréter, actualiser. Pourquoi les études littéraires ?, op. cit., pp. 209-213.

(21) Yves Citton cite entre autres : Patrick Demougin et Jean-François Masso (dir.), Lecture privée et lecture scolaire: la question de la littérature à l'école, Grenoble, CRDP, 1999 ; Annie Rouxel et Gérard Langlade, Le Sujet Lecteur. Lecture subjective et enseignement de la littérature, Rennes, Presses universitaires de Rennes, 2004 ; Jean-Louis Dufays, Louis Gemmenne et Dominique Ledur, Pour une lecture littéraire. Histoire, théories, pistes pour la classe, Bruxelles, de Boeck, $2^{\mathrm{e}}$ édition, 2005 ; Anick Brillant-Annequin et Jean-François Massol, Le Pari de la littérature: quelles littératures de l'école au lycée ?, Grenoble, CRDP, 2005.

(22) Yves Citton, Lire, interpréter, actualiser. Pourquoi les études littéraires?, op. cit., p. 209. 
- « si nous voulons favoriser et enrichir les processus d'individuation symbolique qui permettent à chacun de constituer, de renforcer et de raffiner sa singularité »;

- « si nous voulons cultiver des processus de participation démocratique capables de donner lieu à une acculturation commune s'enrichissant de façon conviviale des singularités qu'elle cultive »;

- «si nous voulons mener, à partir du mode d'interaction régissant l'espace de nos salles de classe, des politiques émancipatrices basées sur le postulat de l'égalité des intelligences - politiques qui constituent le meilleur moyen de renforcer et d'affiner notre nationalité collective »;

- «si nous voulons favoriser le développement d'une virtuosité improvisatrice qui devient de plus en plus utile et nécessaire avec l'accroissement de complexité de nos modes d'interactions sociales, en ce qu'elle permet un ajustement en temps réel de la pensée à ses conditions immédiates de diffusion et de production collective ${ }^{(23)} »$.

Raymond Michel, CELTED, Université Paul Verlaine, Metz.

\section{Table ronde - Recherche et didactique}

\section{Quelle articulation entre la formation des maîtres et la recherche en didactique du français?}

J'aborderai la question de la didactique du français et de la recherche à partir du problème qui occupe mon temps et ma réflexion depuis presque deux ans, celui de la formation d'enseignants, dans la perspective des master en particulier. Il est constitutif d'un master de comporter une dimension de recherche, et l'idée que la formation des enseignants soit adossée à la recherche est un vieux mot d'ordre, syndical entre autres, une évidence qui paraît partagée, au moins entre nous. Bien sûr l'idée que cette recherche, qui donnerait leur légitimité à des master d'enseignement, soit la recherche en didactique est sans doute moins partagée. Cependant la légitimité d'un adossement à la recherche semble aller de soi. Mais est-ce si évident ? Quelles seraient ses vertus pour la formation d'enseignants, plus spécifiquement celles de la recherche en didactique? Si on ne veut pas que ce soit simplement un slogan, il faut se demander précisément ce qu'on peut en attendre, quelles fonctions lui donner dans une formation d'enseignants.

C'est la question que nous nous sommes posée au moment de concevoir un master qui comporterait un horaire conséquent entrant sous la rubrique recherche, avec le souci que cet investissement éclaire l'ensemble de la formation et soit réellement une ressource pour l'exercice du métier. Dans notre projet, cela consiste classiquement en heures de séminaire destinées à accompagner, à partir du second semestre, les futurs enseignants dans une pratique personnelle de réflexion respectant certains critères de la recherche (problématisation, recueil de données...) sur une des dimensions du métier. Mais le choix a été aussi d'attribuer en première année des heures pour présenter

(23) Ibid., pp. 210, 211, 213, 215, 216, 218, 223 et 227. 